Original research article

\title{
Effect of the natural flavonoids myricetin and dihydromyricetin on the wound healing process in vitro
}

\author{
Renáta Sklenářová ${ }^{1}$, Marika Svrčková ${ }^{1}$, Petr Hodek $^{2}$, Jitka Ulrichová ${ }^{1}$, Jana Franková ${ }^{1}$ * \\ ${ }^{1}$ Palacký University Olomouc, Faculty of Medicine and Dentistry, Department of Medical Chemistry and Biochemistry, Olomouc, Czech Republic \\ ${ }^{2}$ Charles University, Faculty of Science, Department of Biochemistry, Prague 2, Czech Republic
}

\begin{abstract}
Myricetin (MYR) and dihydromyricetin (DHM) are classified as natural flavonoids. Both substances are known for their anti-inflammatory and antioxidant properties. In this study, an in vitro model of inflammation was demonstrated on monolayers of scratched fibroblasts or keratinocytes exposed to LPS from Pseudomonas aeruginosa for six hours. MYR and DHM were subsequently applied to the cells for 24 hours at sub toxic concentrations $(5-15 \mu \mathrm{M})$. Inflammatory parameters were analysed in collected cell medium and lysate after the incubation period using the Enzyme-Linked ImmuneSorbent Assay (ELISA) and Western blot. Both flavonoids inhibit the production of pro-inflammatory cytokines (IL-6, IL-8) in LPS-stimulated skin cells as well as the decreased level of MMP-1 in fibroblasts. However, the application of MYR and DHM dose dependently increased the level of MMP-1 in keratinocytes. In our experiments, we focused on the anti-glycation activity of MYR and DHM, where the higher concentration of MYR seems to be more effective.
\end{abstract}

Keywords: Dihydromyricetin; Inflammation; Myricetin; Skin wound healing

Highlights:

- Myricetin and dihydromyricetin induced a molecular mechanism involved in the wound healing process in vitro.

- Myricetin and dihydromyricetin decreased the selected inflammatory parameters in LPS stimulated dermal human fibroblasts and keratinocytes.

- It was demonstrated that myricetin and dihydromyricetin supported anti-glycation activity.

\begin{abstract}
Abbreviations:
AGEs, Advanced Glycation End Products; MYR, Myricetin; DHM, Dihydromyricetin; NFkB, nuclear factor $\kappa B$; Nrf2, nuclear factor erythroid 2-related factor 2; COX-2, cyclooxygenase-2; MMPs, metalloproteinases; IL-6, interleukin 6; IL-8, interleukin 8; TIMP 1, tissue inhibitor of metalloproteinase 1; MTT, [3-(4,5-dimethyltiazol-2 yl) 2, 5 diphenyltetrazolium bromide]; MAPK, mitogen-activated protein kinase; TNF- $\alpha$, tumor necrosis factor $\alpha$; LPS, lipopolysaccharide; DMEM, Dulbecco's modified Eagle's medium; FBS, fetal bovine serum; EPI, EpiLife growth medium; KBM-2, Keratinocyte basal medium; HKGS, Human Keratinocyte Growth Supplement kit; NHDFs, Normal human dermal fibroblasts; NHEKs, Normal human epidermal keratinocytes; $C$, control untreated cells; CS, untreated scratched cells; CL, untreated scratched cells with LPS; PBS, phosphate buffered saline; TBS/T, Tris-buffered saline with 0,05\% Tween 20; BSA, Bovine Serum Albumin; PVDF membrane, Polyvinylidene fluoride membrane; TMB substrate, 3,3',5,5'-Tetramethylbenzidine substrate; ABTS substrate, [2,2'-azino-bis(3-ethylbenzothiazoline-6-sulphonic acid)] substrate
\end{abstract}

\section{Introduction}

Wound healing is a complex system consisting of inflammation, cellular proliferation and remodelling of the extracellular matrix (Takeo et al., 2015). Although these phases play a key role in the physiological process of wound healing, persistent inflammation may cause prolongation of the wound healing process and damage healthy tissue (Valluru et al., 2011; Zhao et al., 2016). Healing complications are characterised by an excess of bacteria, massive biofilm formation, overproduction of ROS and overexpression of MMPs that consequently increase the production of high amounts of pro-inflammatory cytokines. LPS from the gram-negative bacteria Pseudomonas aeruginosa is well known as a potent stimulator of inflammatory cytokines that ultimately induce connective tissue breakdown. This inducer of inflammation is able to interact with Toll-like receptors and stimulate the secretion of pro-inflammatory cytokines (e.g. IL-6 and IL- 8) (Arranz-Valsero et al., 2014; Wang et al., 2003). It plays an important role in the activation of the nuclear factor (erythroid-derived 2)-like 2 (Nrf2) and nuclear factor kappa B (NF- $\mathrm{B})$ signalling pathways. $\mathrm{NF}-\mathrm{kB}$ and Nrf2 are the two key transcription factors that regulate cellular responses to oxidative stress and inflammation

\footnotetext{
* Corresponding author: Jana Franková, Palacký University Olomouc, Faculty of Medicine and Dentistry, Department of Medical Chemistry and Biochemistry, Hněvotínská 3, 77515 Olomouc, Czech Republic; e-mail: jana.frankova@upol.cz http://doi.org/10.32725/jab.2021.017

Submitted: 2020-11-18 • Accepted: 2021-07-20 • Prepublished online: 2021-08-10

J Appl Biomed 19/3: 149-158 • EISSN 1214-0287 • ISSN 1214-021X

(c) 2021 The Authors. Published by University of South Bohemia in České Budějovice, Faculty of Health and Social Sciences.

This is an open access article under the CC BY-NC-ND license.
} 
respectively. Their activity is essential for maintaining and coordinating the cellular responses that resolve the inflammatory status of the cell/tissue (Wardyn et al., 2015). Pro-inflammatory cytokines cause degradation of the I $\kappa \mathrm{B}$ inhibitor. NF- $\kappa \mathrm{B}$ can be translocated to the nucleus to start the expression of target genes and activate the signal pathway that triggers the production of AGEs (Khan et al., 2018; Liu et al., 2018). Thus, AGEs can inhibit the proliferation of fibroblasts and keratinocytes via the transduction NF- $\kappa B$ signal (Qing, 2017).

One possible method to regulate the healing process is the therapeutic use of natural compounds such as flavonoids. They are studied due to their anti-inflammatory, antioxidant, anti-cancer, anti-photoaging, and immunomodulatory activity (Jing and Li, 2019; Ma et al., 2019; Xie and Zheng, 2017). They are also a significant source of AGE inhibitors. In this study, two flavonoids were selected: the plant secondary metabolites MYR and DHM. These flavonoid compounds have low toxicity and differing pharmacological properties. Although MYR and DHM differ only in the double bond between C2 and C3 on the flavon ring (Fig. 1), MYR, which is found in many foods and fruits and its important natural producer is also Myrica nagi (esculenta) (Preeti Panthari et al., 2012), has been researched more thoroughly to date. The anti-inflammatory activity of MYR has been demonstrated in a variety of in vitro assays, where the inhibition of pro-inflammatory cytokines and MMPs was evaluated. Further, MYR prevents activation of

(A) Myricetin (MYR)<smiles>O=c1c(O)c(-c2cc(O)c(O)c(O)c2)oc2cc(O)cc(O)c12</smiles>

the NF- $\kappa B$ signalling pathway (Grenier et al., 2015). Elshamy et al. (2020) recently published a study carried out on albino rats. The rats were injured and treated with $10 \%$ and $20 \%$ MYR for 14 days to indicate its healing properties. Treatment with $20 \%$ MYR increased the percentage of wound closure, reduced scarring and induced reepithelialization with a high density of fibroblasts and blood capillaries.

DHM is a plant flavonoid isolated from Hovenia dulcis or Ampelopsis grossedentata and is often used in traditional Chinese medicine (Zhang et al., 2014). Previously, Hovenia dulcis extract has been extensively investigated for its effects in the suppression of acute EtOH intoxication (Shen et al., 2012). Recent studies of DHM have dealt with its cardioprotective, anti-cancer and antibacterial properties (Liang et al., 2019; Shevelev et al., 2020; Sun et al., 2020, Wu et al., 2017; 2018).

Despite the limited available knowledge of the effect of DHM on the mechanisms of the wound healing process, it has emerged as a promising substance for the treatment of inflammation. The present study evaluated the anti-inflammatory and anti-glycation effect of MYR and DHM on an in vitro model of scratched monolayers of normal human dermal fibroblasts (NHDFs) and normal human epidermal keratinocytes (NHEKs) pre-treated with LPS (P. aeruginosa). The selected pro-inflammatory cytokines, production of AGEs, MMPs, as well as NF- $\mathrm{B}$, Nrf2, COX-2 and MMP-2 were clarified.

(B) Dihydromyricetin (DHM)<smiles>O=C1c2c(O)cc(O)cc2O[C@H](c2cc(O)c(O)c(O)c2)[C@@H]1O</smiles>

Fig. 1. The structure of myricetin (A) and dihydromyricetin (B)

\section{Materials and methods}

\section{Chemicals}

DHM (APIChem, 98\% purity, People's Republic of China, Hangzhou APIChem Technology Co., Ltd.) and MYR (TCI, Japan, 97\% purity, Tokyo Chemical Industry Co., Ltd.) were tested flavonoids. DMEM, FBS, ATB (penicillin, streptomycin, ampicillin), EPI, KBM 2 with KGM-2, HKGS kit and LPS from Pseudomonas aeruginosa were used for cell culture.

Polyclonal antibodies that were used in Western blot: rabbit anti-MMP-2 (sc-10736), rabbit anti-Nrf2 (sc 722), rabbit anti COX-2 (sc-7951), rabbit anti-NF-kB (sc-372) and goat anti- $\beta$-actin (sc 1616) were purchased from Santa Cruz Biotechnology. ELISA Human IL-6 Kit (PeproTech), ELISA Human IL-8 Kit (PeproTech), Human Total MMP-1 DuoSet ELISA (R\&D Systems), Human MMP-2 DuoSet ELISA (R\&D Systems), Human TIMP-1 DuoSet ELISA (R\&D Systems) and TMB substrate, streptavidin-HRP, Avidin-HRP, ABST substrate were used to detect MMPs and interleukins (IL-6, IL-8) by ELISA. DMSO+NH 3 , MTT (3-(4,5-dimethyltiazol-2 yl) 2, 5 diphenyltetrazolium bromide) were used to measure cell viability. Methylglyoxal and aminoguanidine were used to determine anti-glycation activity.

\section{Cell culture}

Normal human dermal fibroblasts (NHDFs) and Normal human epidermal keratinocytes (NHEKs) were obtained from healthy tissue samples taken from plastic surgery patients from Faculty Hospital Olomouc after informed consent. The study was performed according to the Code of Ethics of the World Medical Association.

NHDFs were cultivated in DMEM with $10 \%$ FBS and antibiotics ( $1 \%$ penicillin-streptomycin) under standard conditions $\left(5 \% \mathrm{CO}_{2}, 37^{\circ} \mathrm{C}\right)$. For the experiments, NHDFs were cultured in serum free medium (DMEM containing $1 \%$ penicillin-streptomycin).

NHEKs were cultivated in KBM-2 with KGM-2 supplements for 3 days under standard conditions $\left(5 \% \mathrm{CO}_{2}, 37^{\circ} \mathrm{C}\right)$. After this period, the medium was exchanged for medium EPI containing HKGS and antibiotics (1\% penicillin-streptomycin, ampicillin) (Franková et al., 2016).

\section{Cell viability assay}

Cell viability was assessed using an MTT assay (Kumar et al., 2018). NHDFs were isolated from tissue section from six patients. The cells were seeded in 96 well plates at $0.2 \times 10^{4}$ cells/ well. As soon as cells reached confluence, they were washed with PBS. MYR and DHM were diluted at 9 concentrations 
(2.5-100 $\mu \mathrm{M})$ in the serum-free DMEM and applied to cells for $24 \mathrm{~h}$. Then, the plates were washed with PBS and incubated with MTT solution for $4 \mathrm{~h}$. The MTT mixture was poured out and a solution containing DMSO $+\mathrm{NH}_{3}$ was added. Absorbance was measured at $540 \mathrm{~nm}$.

\section{Treatment experiment}

The cells were used at the third passage and seeded on Petri dishes $(10 \mathrm{~cm})$. When the cells reached confluence, they were scratched with $10 \mathrm{ml}$ plastic pipettes. LPS from Pseudomonas aeruginosa (final concentration $10 \mu \mathrm{g} / \mathrm{ml}$ ) was added for $6 \mathrm{~h}$ to stimulate the pathological inflammatory response (Juráňová et al., 2019). For controls, only cells with the cultivation medium (without scratch) (C), untreated scratched cells (CS) and untreated scratched cells with LPS (CL) were used. A 100 mM solution of MYR and DHM in DMSO was prepared and individual samples were diluted in serum free medium to final concentration $(5 \mu \mathrm{M}, 10 \mu \mathrm{M}$ and $15 \mu \mathrm{M})$. After the pre incubation period with LPS, MYR and DHM were applied for $24 \mathrm{~h}$. At the end of the incubation period, the cell culture medium was collected, and the cells were lysed with RIPA buffer supplement with protease inhibitors and stored at $-80^{\circ} \mathrm{C}$.

\section{Western blot analysis}

Western blot analysis was used for the detection of transcription factors, such as NF- $\mathrm{BB}$ and Nrf2, as well as parameters connected to inflammation (COX 2 and MMP 2). $\beta$-actin was selected as a reference protein and protein level in the cell lysate was quantified by the Bradford method (Bradford, 1976). The cell lysate was diluted $4: 1$ with Treatment sample buffer. Subsequently, the sample was boiled for $5 \mathrm{~min}$ and cooled on ice. It was tempered to $37^{\circ} \mathrm{C}$ before electrophohesis. Equal amounts of protein cell lysates $(40 \mu \mathrm{g})$ were subjected to SDS-PAGE in $10 \%$ polyacrylamide gel. Then, the proteins were transferred to PVDF membranes, blocked in 5\% low-fat milk in TBS/T or in 5\% BSA in TBS/T (MMP-2) for $2 \mathrm{~h}$ at room temperature. The primary antibodies were diluted $1: 500$ and incubated overnight at $4{ }^{\circ} \mathrm{C}$. The following day, the membranes were washed in TBS/T for $30 \mathrm{~min}$ and secondary antibodies (diluted 1 : 10 000) were subsequently applied for $1.5 \mathrm{~h}$ before the membranes were washed again. A chemiluminescent blotting substrate was used for detection and the intensity of the bands on the films was quantified by densitometry analysis using ImageJ (Juráňová et al., 2019).

\section{Detection of IL-6 and IL-8}

ELISA Human IL-6 Kit and ELISA Human IL-8 Kit were used for analyses of IL 6 and IL- 8 in the cell culture medium after the treatment as per the manufacturer's protocol. Briefly, the plates were coated with capture antibody diluted in PBS and incubated overnight at room temperature. The following day, the plates were washed and incubated with Block buffer for $2 \mathrm{~h}$. After the incubation period, standards and samples were added. After $2 \mathrm{~h}$, the plates were washed, and detection antibodies were applied. After washing, Avidin-HRP was applied for $30 \mathrm{~min}$ and then the plates were washed again. Finally, substrate solution (ABST) was added and absorbance was measured at $405 \mathrm{~nm}$ with wavelength correction set at $650 \mathrm{~nm}$ using an ELISA 96 - plate reader.

\section{Detection of MMP-1, MMP-2 and TIMP-1}

Human Total MMP-1 DuoSet ELISA, Human MMP-2 DuoSet ELISA, and Human TIMP-1 DuoSet ELISA were used to analyse MMP-1, MMP-2 and TIMP-1 in cell culture medium according to the manufacturer's protocol. Briefly, the capture antibody was diluted to the working concentration in PBS and pipetted onto 96-well plates. The plates were incubated overnight at room temperature. Subsequently, the plates were washed with Wash buffer and blocked with Reagent diluent. In the next step, standards and samples were added and incubated for $2 \mathrm{~h}$. After the incubation period, the plates were washed and detection antibodies were pipetted into each well. The plates were washed after $2 \mathrm{~h}$, Streptavidin-HRP was applied for $20 \mathrm{~min}$ and they were washed again. Substrate solution (TMB) was added and, after $20 \mathrm{~min}$, the reaction was stopped with sulphuric acid solution ( $2 \mathrm{M} \mathrm{H}_{2} \mathrm{SO}_{4}$ ) and absorbance was measured at $450 \mathrm{~nm}$ with wavelength correction set at $540 \mathrm{~nm}$.

\section{Detection of anti-glycation activities by fluorescence spectroscopy}

Fluorescence spectroscopy is a widely used method for the identification and measurement of glycation or anti-glycation activities. This method is based on the detection of the intrinsic fluorescence of AGEs within the protein structure at the ex/em wavelengths $350 / 420 \mathrm{~nm}$. For the purposes of this study, fluorescence intensities of $5 \mathrm{mg} / \mathrm{ml} \mathrm{BSA} \mathrm{samples} \mathrm{in}$ $0.1 \mathrm{M}$ PBS (pH 7.4) were evaluated at $420 \mathrm{~nm}$ after excitation at $350 \mathrm{~nm}$, using a fluorescence microplate reader (Infinite M200 PRO, Tecan) operating at room temperature (integration time $20 \mu \mathrm{s}$; excitation bandwidth $9 \mathrm{~nm}$; emission bandwidth $20 \mathrm{~nm}$ ). For more details, see Yanagisawa et al. (1998).

The final concentration of BSA was $5 \mathrm{mg} / \mathrm{ml}$, glycation was performed by the incubation of BSA in the presence of $100 \mu \mathrm{M}$ methylglyoxal for $48 \mathrm{~h}$ at $37^{\circ} \mathrm{C}$. Incubation of a sample in the presence of $100 \mu \mathrm{M}$ aminoguanidine was used as the control.

\section{Statistical analysis}

Unless stated otherwise, the measurements were carried out in five independent experiments with different cell donors and were expressed as the mean \pm SEM.

Statistical differences were determined using the Student's $t$ test. Values of ${ }^{*} P<0.05,{ }^{* *} P<0.01$ and ${ }^{* * *} P<0.001$ were considered significant.

\section{Results}

\section{Cell viability assay}

Cell viability was evaluated by MTT assay, which measured the mitochondrial reduction of MTT [3-(4,5-dimethyltiazol2-yl)-2, 5-diphenyltetrazolium bromide] to insoluble purple formazan.

On the basis of the MTT assay, sub-toxic concentrations were chosen (5, 10 and $15 \mu \mathrm{M}$ MYR and DHM). The higher concentrations were considered to be toxic, e.g. $20 \mu \mathrm{M}$ concentration caused a $46 \%$ decrease in cell viability with MYR and $24 \%$ with DHM and concentrations in the range from $30 \mu \mathrm{M}$ to $100 \mu \mathrm{M}$ MYR, and DHM caused a $70 \%$ decrease in cell viability (data not shown).

\section{Western blot analysis}

Western blot analysis was used to evaluate if the treatment of MYR and DHM regulated the expression of inflammatory proteins and transcription factors (COX-2, Nrf2, NF- $\kappa$ B and MMP-2) in the cell lysate of fibroblasts and keratinocytes (Figs. 2 and 3 ). 
A.

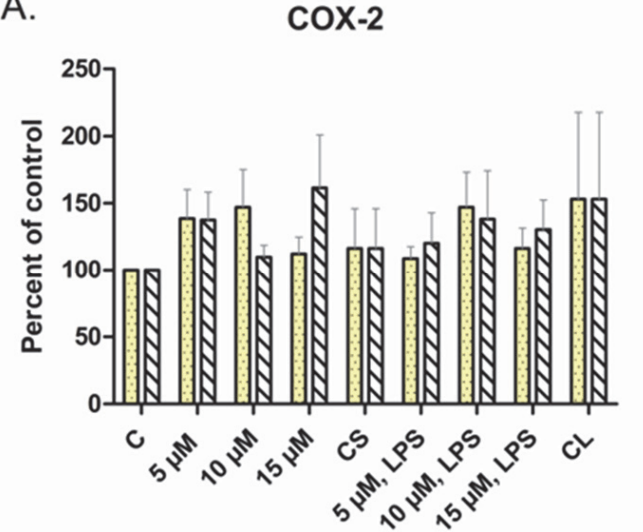

C.

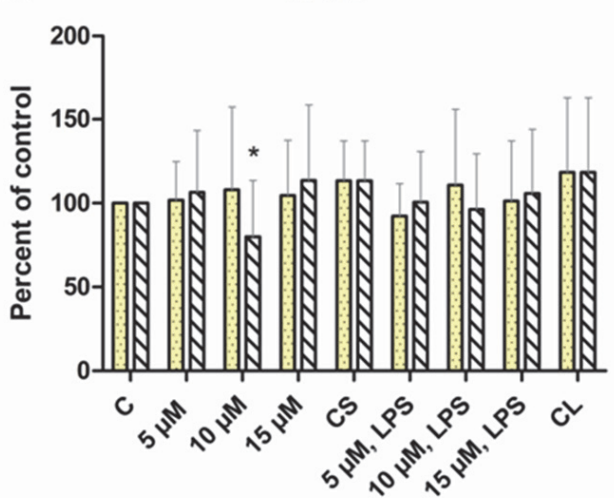

B.

Nrf2

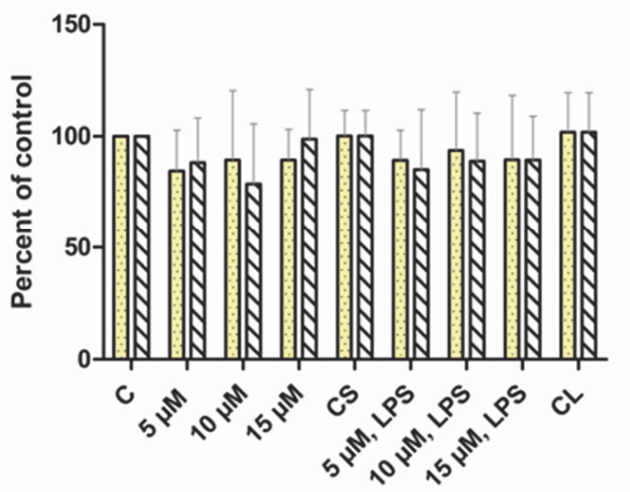

MYR

ब $\mathrm{DHM}$

E.

D.

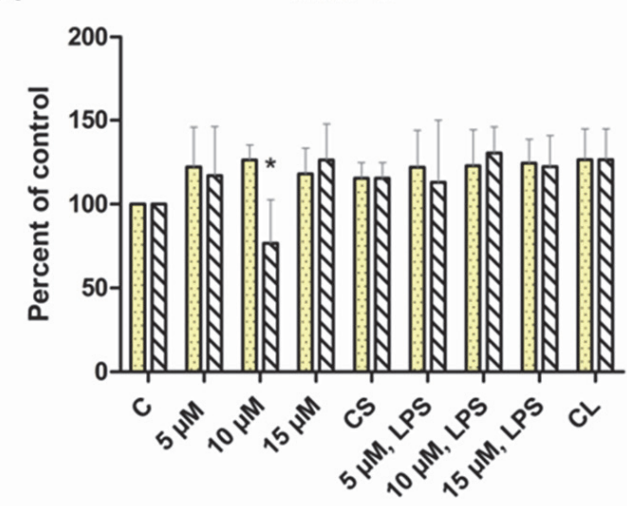

罾 MYR $\triangle \mathrm{DHM}$

Fig. 2. The effects of MYR and DHM on the expression of (A) COX-2, (B) Nrf2, (C) NF- $\mathrm{BB},(\mathbf{D})$ MMP-2 determined by Western blot in cell lysate of NHDFs. Representative Western blot $(\mathbf{E}) .{ }^{*} P<0.05$ were considered significant compared to the scratch control (CS). The control (C) was NHDFs that were not scratched and treated. The other controls were untreated scratched cells (CS) and untreated scratched cells with LPS (CL). Number of measurements: $n=5$.

Analysis of the selected flavonoids used to inhibit the inducible pro-inflammatory enzyme COX-2 showed that its level was increased in scratched NHDFs, but only treatments of $15 \mu \mathrm{M}$ MYR and $10 \mu \mathrm{M}$ DHM decreased the level of COX-2 compared to the CS. On the other hand, the level of COX-2 was decreased at all tested concentrations of MYR and DHM in LPS stimulated NHDFs compare to the CL. Further, COX-2 levels were reduced in a dose dependent manner by MYR and DHM in NHEKs (Fig. 2A, 3A).

Next, we examined the antioxidant activity of MYR and DHM and their effect on the Nrf2 signalling pathway. The activation and repression of Nrf2 provides protection against oxidative stress. In our study, we found that the level of the transcription factor Nrf2 was decreased in scratched NHDFs and NHEKs for all tested concentrations of MYR and DHM compared to the CS. Likewise, DHM and MYR diminished the level of Nrf2 in LPS-stimulated NHDFs and NHEKs compared to the CL (Fig. 2B, 3B).

$\mathrm{NF}-\kappa \mathrm{B}$ is a transcription factor that is implicated in the initiation of pro-inflammatory target gene expression. We determined its level in scratched NHDFs and NHEKs and, surprisingly, it was significantly decreased by a $10 \mu \mathrm{M}$ concentration of DHM compared to the CS. The other tested concentrations of MYR and DHM did not significantly affect the level of 
NF- $\kappa B$ in scratched cells. Concurrently, treatment with MYR and DHM resulted in a slight reduction in the level of NF- $\kappa B$ in LPS-stimulated NHDFs compared to the CL (Fig. 2C, 3C).

Western blot results revealed that $10 \mu \mathrm{M}$ DHM significantly decreased the level of MMP-2 in scratched NHDFs and
NHEKs compared to the CS. For the other tested concentrations applied to NHDF, the level of MMP-2 was identical to the control CL and CS. Concurrently, MMP-2 levels decreased with increasing concentrations of MYR and DHM in LPS-stimulated NHEKs compared to the CL (Fig. 2D, 3D).
A.

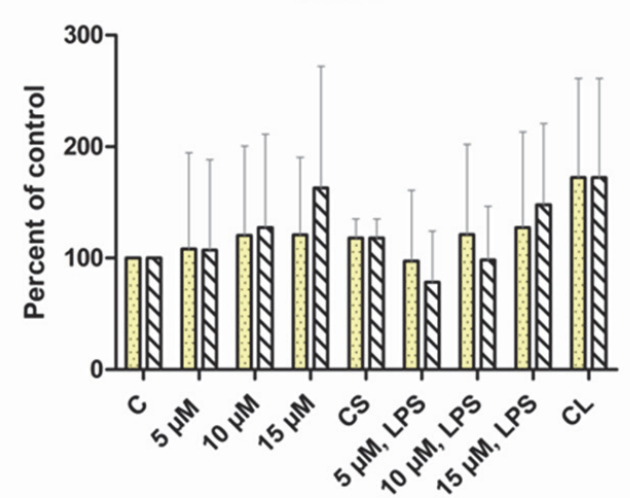

C.

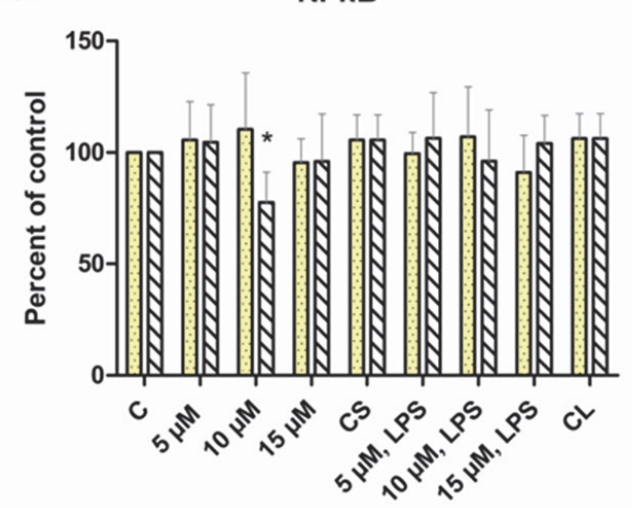

B.

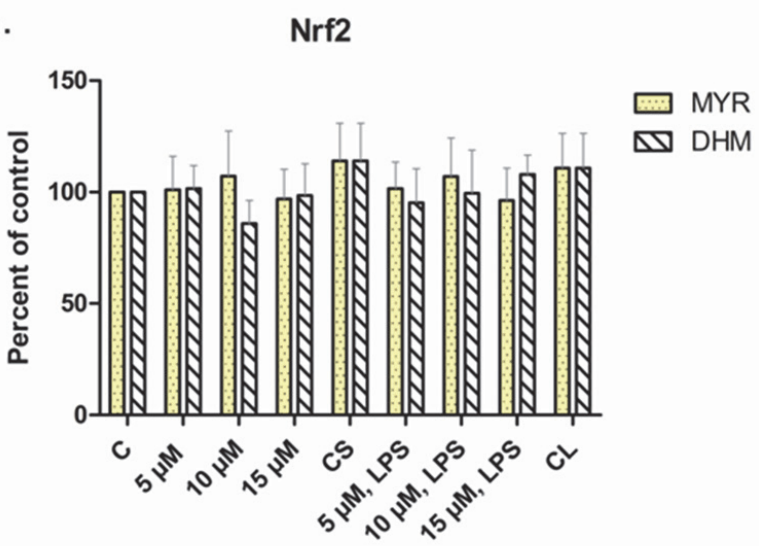

D.

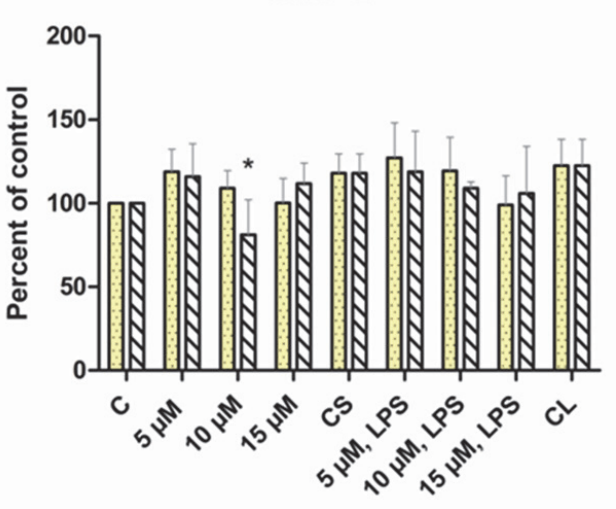

罚 MYR $\triangle \mathrm{DHM}$ DHM

E.

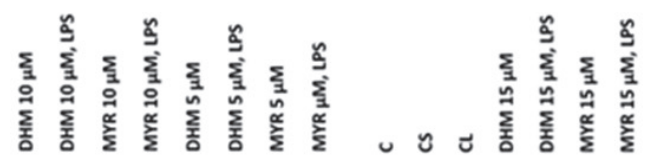
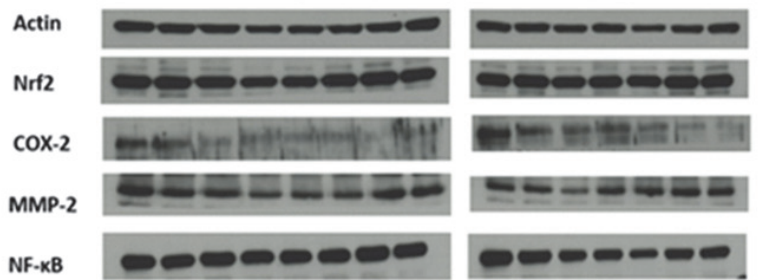

Fig. 3. The effects of MYR and DHM on the expression of (A) COX-2, (B) Nrf2, (C) NF-kB, (D) MMP-2 determined by Western blot in cell lysate of NHEKs. Representative Western blot $(\mathbf{E}){ }^{*} P<0.05$ were considered significant compared to the scratch control (CS). The control (C) was NHEKs that were not scratched and treated. The other controls were untreated scratched cells (CS) and untreated scratched cells with LPS (CL). Number of measurements: $n=5$. 


\section{Enzyme-Linked ImmunoSorbent Assay (ELISA) Determination of IL-6 and IL-8}

A.

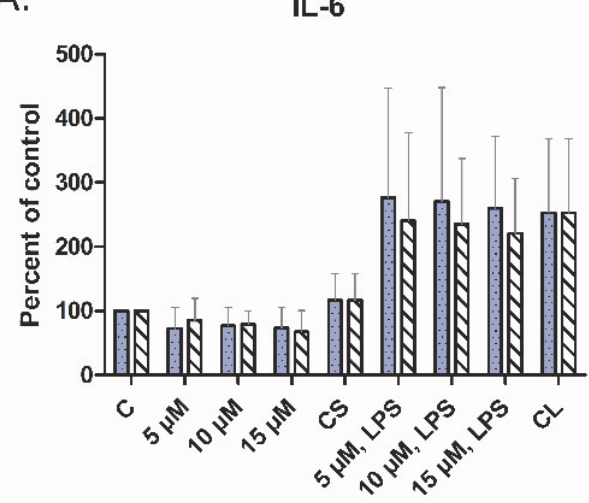

C.

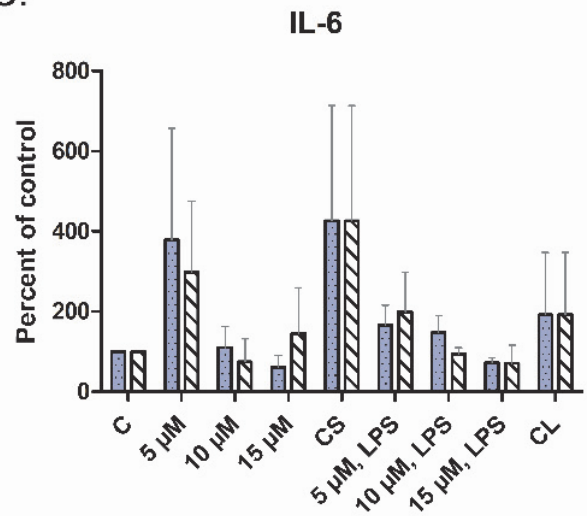

B.

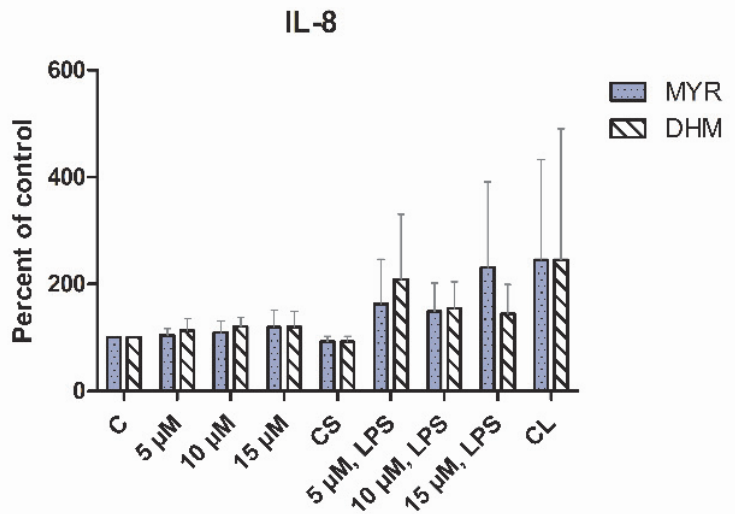

D.

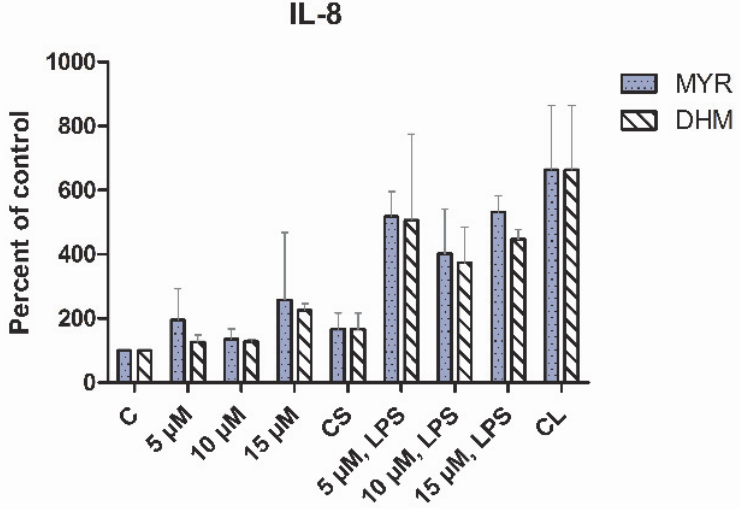

Fig. 4. The effects of MYR and DHM on IL-6 (A) and IL-8 (B) production by NHDFs and IL-6 (C) and IL-8 (D) production by NHEKs. The control (C) was cells that were not scratched and treated. The other controls were untreated scratched cells (CS) and untreated scratched cells with LPS (CL). Number of measurements: $n=5$.

To evaluate the inhibitory effects of MYR and DHM on inflammatory cytokines and chemokines of human skin cells, IL-6 and IL-8 were measured using ELISA kits. The level of IL-6 was lower after a 24 hours treatment with MYR and DHM in scratched NHDFs compared to the CS. On the other hand, the level of IL-8 was raised compared to the CS. In addition, treatment with MYR and DHM resulted in a slight reduction in pro-inflammatory cytokines production in LPS-stimulated NHDFs (Fig. 4A, 4B).

Cytokine IL-6 levels were lessened in a dose dependent manner by MYR and DHM in scratched and LPS-stimulated NHEKs. Further, MYR and DHM also diminished the level of chemokine IL-8 in LPS-stimulated NHEKs compared to the CL (Fig. 4C, 4D).

In cutaneous wounds, MMPs are synthesized by keratinocytes, fibroblasts, macrophages, and endothelial cells and are upregulated within hours after injury. In our study, we observed different reactions in the secretion of MMPs after MYR and DHM treatments of skin cells. The level of MMP-1 decreased with increasing concentrations of MYR and DHM in scratched and LPS-stimulated NHDFs (Fig. 5A).

For all selected concentrations of MYR and DHM, the level of MMP-2 was higher in scratched NHDFs compared to the CS. MMP-2 in LPS-stimulated NHDFs was intensified compared to the $\mathrm{CL}$ after treatment with MYR; nevertheless 10 and $15 \mu \mathrm{M}$ concentrations of DHM reduced the level of MMP-2 compared to the $\mathrm{CL}$ (Fig. 5B).

The selected flavonoids affected TIMP production. It was observed that MYR and DHM at concentrations of 5 and $15 \mu \mathrm{M}$ increased TIMP-1 in scratched NHDFs compared to the CS. Further, in LPS-stimulated NDHFs, the production of TIMP-1 at all concentrations of MYR and DHM was comparable to the $\mathrm{CL}$ (Fig. 5C).

NHDFs and NHEKs produced matrix metalloproteinases (MMPs) and tissue inhibitors of metalloproteinases (TIMPs) at differing intensities. The secretion of collagenase-1 (MMP-1) was greater after the treatment by MYR and DHM in NHEKs compared to NHDFs. Its level increased relative to increasing concentrations of MYR and DHM in scratched and LPS-stimulated NHEKs (Fig. 6A).

On the other hand, the production of MMP-2 was diminished by treatment with MYR and DHM in scratched NHEKs compared to the CS. In addition, the level of MMP-2 in LPS-stimulated NHEKs was increased at all tested concentrations except $10 \mu \mathrm{M}$ MYR compared to the CL (Fig. 6B).

The level of TIMP-1 was increased in a dose dependent manner when treated with MYR and DHM in scratched NHEKs compared to the CS. Stimulation of LPS-treated NHEKs with MYR induced secretion of TIMP-1. The level of TIMP-1 rose with increasing concentrations of MYR in LPS-stimulated 
A.

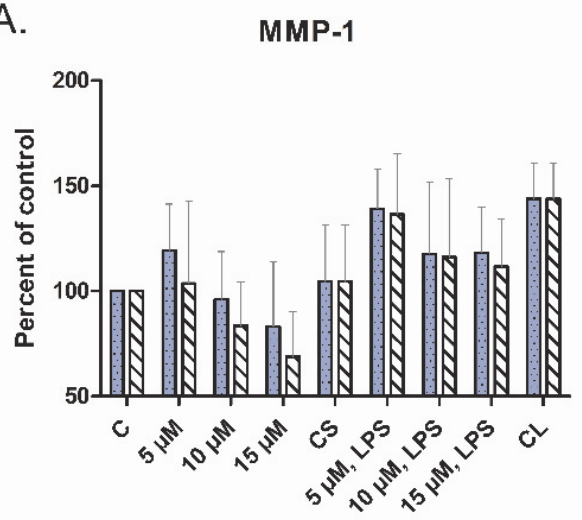

B.

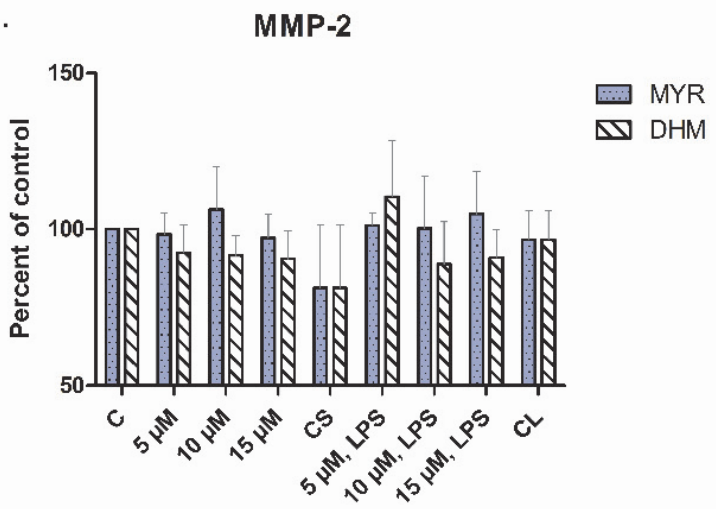

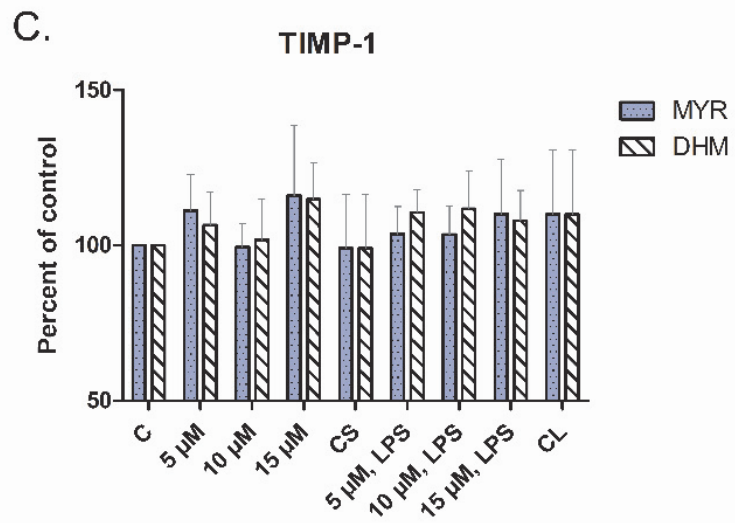

Fig. 5. The effects of MYR and DHM on MMP-1 (A), MMP-2 (B) and TIMP-1 (C) production by NHDFs. The control (C) was NHDFs that was not scratched and treated. The other controls were untreated scratched cells (CS) and untreated scratched cells with LPS (CL). Number of measurements: $n=5$.

NHEKs. However, TIMP-1 level was reduced with increasing concentrations of DHM in LPS-stimulated NHEKs compared to the $\mathrm{CL}$ (Fig. 6C).

\section{Anti-glycation activities}

The dependence of BSA glycation rates on the concentration of MYR and DHM is shown in Fig. 7. Glycated BSA in the absence of MYR / DHM was set as 1. For example, $50 \mu \mathrm{M}$ MYR reduced the glycation rate by almost $30 \%$, while $50 \mu \mathrm{M}$ DHM reduced the glycation rate by $26 \%$. For MYR and DHM, the specific fluorescence was decreased over $48 \mathrm{~h}$ of methylglyoxal mediated BSA glycation at $37{ }^{\circ} \mathrm{C}$ in a dose-dependent manner up to $50 \mu \mathrm{M}$. For the control, for samples where the methylglyoxal glycation of BSA was suppressed with $10 \mathrm{mM}$ aminoguanidine, the protein fluorescence signal was completely unchanged, i.e. equal to $100 \%$ of the original fluorescence signal (data not shown).

\section{Discussion}

Wound healing is comprised of a cascade of biochemical processes, with three main phases - inflammation, reepithelization and tissue remodelling (Schreml et al., 2010). Based on recent studies, it is obvious that extension of the inflamma- tion phase gives rise to non-healing wounds. This presents considerable problems in wound care and it is therefore appropriate to regulate pathological inflammation. One possible way to manage it is the therapeutic use of natural substances. In this study, the effects of natural flavonoids such as MYR and DHM on LPS-stimulated scratched NHDFs and NHEKs were analysed. These flavonoids, with six hydroxyl groups, are known for their strong antioxidant effect. Despite their similar structure, some studies have shown MYR to have more pronounced antiviral effects (Zhong et al., 2014). Treatment with DHM also has many benefits, e.g. it could be used to accelerate wound repair during the stages of scar formation, as described in a recent in vivo study (Shevelev et al., 2020). One of the main goals of this study is to compare the effect of these two flavonoids on the regulation of the inflammatory response in vitro.

In order to ascertain whether or not MYR and DHM affect NF-kB and Nrf2 signalling pathways (Lei et al., 2018), the cells were treated with LPS. At the time when a wound was established and treated with LPS, NF- $\kappa \mathrm{B}$ was activated and translocated to the nucleus as p65 NF- $\mathrm{BB}$, where it bound to DNA and activated the transcription of the pro-inflammatory target genes (Alvira, 2014; Ambrozova et al., 2017). Both flavonoids, in a concentration range from 5 to $15 \mu \mathrm{M}$, were effective in suppressing expression of NF- $\kappa B$ in LPS-stimulated 
A.

MMP-1

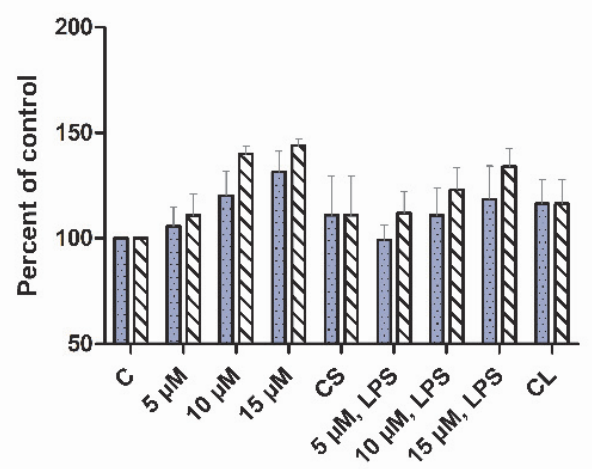

C.
B.

MMP-2

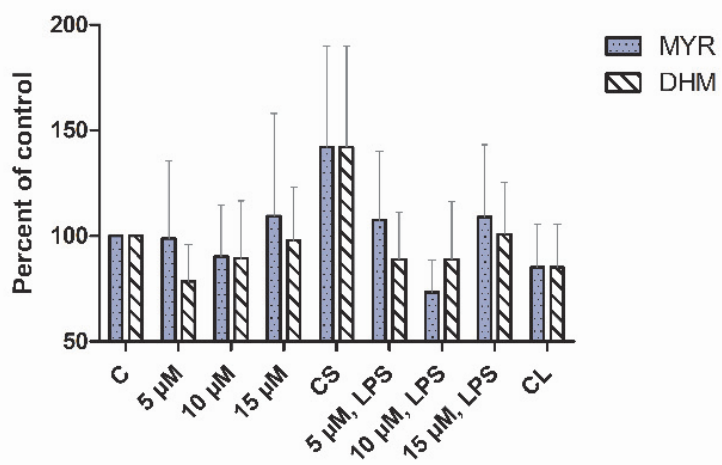

TIMP-1

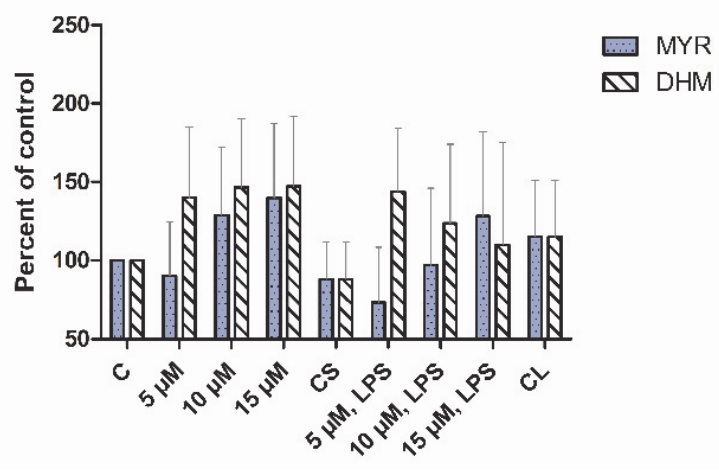

Fig. 6. The effects of MYR and DHM on MMP-1 (A), MMP-2 (B) and TIMP-1 (C) production by NHEKs. The control (C) was NHEKs that were not scratched and treated. The other controls were untreated scratched cells (CS) and untreated scratched cells with LPS (CL). Number of measurements: $n=5$.

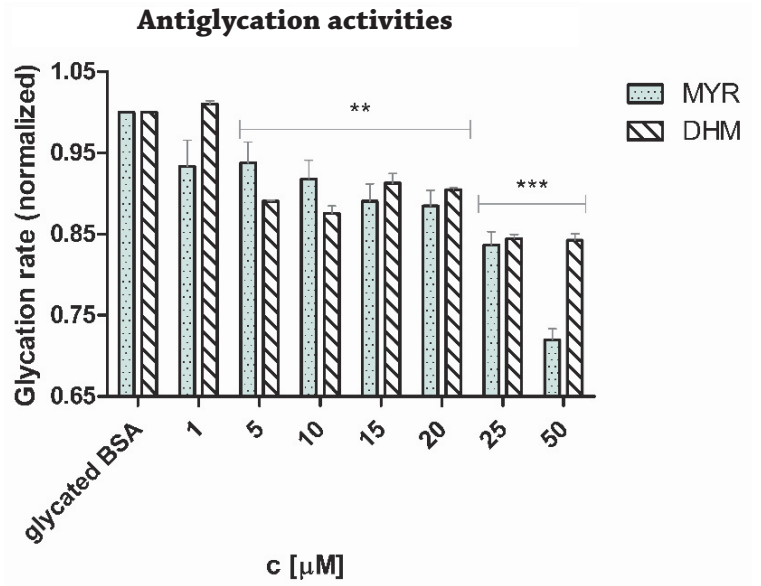

Fig. 7. The effect of MYR and DHM on BSA glycation rate. ${ }^{* *} P<0.01$ and ${ }^{* * *} P<0.001$ were considered significant compared to glycated BSA.

NHDFs and NHEKs compare to the control (CL). Our results demonstrated that DHM at a concentration of $10 \mu \mathrm{M}$ significantly decreased this transcription factor (NF- $\mathrm{kB}$ ) in scratched NHDFs and NHEKs. The fact that DHM could be a potential natural inhibitor of NF- $\mathrm{KB}$ was postulated in an article published by Tang et al. (2016), who found that DHM decreased translocations of p65 NF- $\mathrm{kB}$ into the nucleus in TNF- $\alpha$ stimulated HeLa cells.
This is in line with an article published by Hou et al., who carried out studies on Balb/c and suggest that DHM possesses many biological effects, such as inhibiting the activity of NF- $\mathrm{kB}$, mitogen-activated protein kinase (MAPK), and suppressing the expression of cyclooxygenase- 2 (COX-2) and levels of pro-inflammatory cytokines, e.g. IL-1 $\beta$, IL-6, TNF- $\alpha$ (Hou et al., 2015). Cyclooxygenases represent enzymes that are important for the transformation of arachidonic acid to prostaglandins, which are important markers of inflammation (Surowiak et al., 2014). COX-2, in an induced form, was detected in the scratched cells (also in NDHFs and NHEKs) and in the presence of LPS, compared to the control, confirming stimulation of inflammation. MYR and DHM were effective in the suppression of COX-2 in LPS stimulated NHDFs and NHEKs, respectively. According to more recent studies (Jang et al., 2020; Jing and Li, 2019), pre-treatment with these flavonoids also diminished the secretion of COX-2 in LPS stimulated BV2 microglia. Thus, the present findings further support the potential of MYR and DHM as neuroprotective agents that inhibit inflammation. On the other hand, lower concentrations (e.g. $5 \mu \mathrm{M}$ ) of MYR and DHM caused a pro-inflammatory effect in NHDFs. We speculate that low concentrations of these flavonoids could help to improve the physiological defence of the injury by increasing levels of COX-2 and activating the interleukin cascade. Nrf2 is a key regulator of the expression of pro-inflammatory cytokines and has been shown to inhibit the level of LPS-induced inflammatory cytokine genes (IL-1 $\beta$ and IL 6) (Kobayashi et al., 2016). On the other hand, the transcription factor Nrf2 primarily regulates the expression of antioxidant genes (Ahmed et al., 2017). In addi- 
tion, in vivo studies have recently been published in which MYR tested on chondrocytes demonstrated the capability of MYR to suppress the NF- $\kappa B$ signalling pathway through the Nrf2/HO-1 axis mediated Nrf2 signalling pathway (Liao et al., 2019; Pan et al., 2019). In our study, the expression of Nrf2 was decreased in NHDFs and NHEKs after treatment with MYR and DHM at all tested concentrations.

Many studies have shown that cytokines, especially inflammatory cytokines (IL-6 and IL 8) are involved in the regulation of the healing process and play an important role in immune response and tissue repair (Kuhn et al., 2014). Indeed, in our study we observed decreases in the production of IL-6 and IL-8, caused by MYR and DHM respectively. Moreover, the observed inhibitory effect of MYR and DHM on the secretion of both interleukins was dose-dependent. The overexpression of selected cytokines (IL-6 and IL-8) may initiate the production of MMP-1 and MMP-2 that often persist in non-healing wounds. Raised levels of these MMPs may cause the wound healing time to be prolonged and cause damage to healthy tissue (Hayden et al., 2011; Martins et al., 2013). It was found that the level of MMP-1 decreased with increasing concentrations of MYR and DHM in scratched NHDFs compared to the CS. Contrary to the results for NHDFs, the level of MMP-1 increased in a dose dependent manner relative to MYR and DHM levels NHEKs. This discrepancy between two selected in vitro models (scratched NHDFs and NHEKs) could be explained due to different responses to the scratch assay and the different basal levels of individual MMPs in NHDFs and NHEKs, as published by Tandara and Mustoe (2011). So, MMP-1 was induced more strongly in cells that were in contact with collagen type I, such as basal keratinocytes. Raised levels of the MMP-1 enzyme often lead to the activation of gelatinase MMP-2 (Visse and Nagase, 2003). According to our results, the level of MMP-2 was diminished by DHM in scratched NHEKs compared to the CS. Interestingly, with decreasing concentrations of DHM, the effect was stronger. In contrast, the level of MMP-2 was increased in scratched NHDFs after treatment with DHM and MYR compared to the CS. The activity of MMP-1 is also regulated by tissue inhibitors of metalloproteinase, e.g. TIMP-1. The level of TIMP-1 increased in a dose dependent manner relative to MYR and DHM levels in scratched NHEKs compared to the CS, which was in line with the anti-inflammatory potential of the tested flavonoids.

Glycation is a non-enzymatic reaction between proteins and reducing saccharides that results in the generation of AGEs. It is obvious that the process of anti-glycation is considered useful for slowing down human ageing, disease recovery and wound healing, because an accumulation of AGEs in the extracellular matrix causes abnormal cross-linking and results in reducing the elasticity of tissues (Hwang et al., 2018; Yeh et al., 2017). The anti-glycation activity of MYR was described by Urios et al. (2007), who wrote that MYR was more effective in the regulation of Pentosidine production than Quercetin. The effect of MYR was also descibed in a later study, which affirmed that flavonoids with 3', 4', 5, 7-hydroxyl substituents demonstrate stronger AGE inhibition (Patil et al., 2019). In our study, we observed that the anti-glycation activity of MYR at higher concentrations seems to be more effective than DHM.

\section{Conclusions}

In conclusion, our experiments demonstrated that the flavonoids MYR and DHM decreased the selected inflammatory parameters in LPS stimulated human fibroblasts and keratino- cytes. The outcomes of the present investigation showed that MYR and DHM supressed the production of AGEs. Based on these results, we suggest that these flavonoids could support the wound-healing process.

\section{Conflict of interests}

The authors declare no conflict of interests.

\section{Acknowledgements}

This work was supported by the grants IGA_LF_2021_011, IGA_LF_2019_015 and P303/12/G163 from the Czech Science Foundation (GACR).

\section{References}

Ahmed SM, Luo L, Namani A, Wang XJ, Tang X (2017). Nrf2 signaling pathway: Pivotal roles in inflammation. Biochim Biophys Acta Mol Basis Dis 1863(2): 585-597. DOI: 10.1016/j. bbadis.2016.11.005

Alvira CM (2014). Nuclear factor-kappa-B signaling in lung development and disease: One pathway, numerous functions. Birth Defects Res A Clin Mol Teratol 100(3): 202-216. DOI: $10.1002 /$ bdra.23233.

Ambrozova N, Ulrichova J, Galandakova A (2017). Models for the study of skin wound healing. The role of Nrf2 and NF-кB. Biomed Pap Med Fac Univ Palacky Olomouc Czech Repub 161(1): 1-13. DOI: $10.5507 / \mathrm{bp} .2016 .063$.

Arranz-Valsero I, Soriano-Romaní L, García-Posadas L, LópezGarcía A, Diebold Y (2014). IL-6 as a corneal wound healing mediator in an in vitro scratch assay. Exp Eye Res 125: 183-192. DOI: 10.1016/j.exer.2014.06.012.

Bradford MM (1976). A rapid and sensitive method for the quantitation of microgram quantities of protein utilizing the principle of protein-dye binding. Anal Biochem 72: 248-254. DOI: 10.1006/abio.1976.9999.

Elshamy AI, Ammar NM, Hassan HA, El-Kashak WA, Al-Rejaie SS, Abd-ElGawad AM, Farrag A-RH (2020). Topical Wound Healing Activity of Myricetin Isolated from Tecomaria capensis v. aurea. Molecules 25(21): 4870. DOI: 10.3390/molecules25214870.

Franková J, Pivodová V, Vágnerová H, Juránová J, Ulrichová J (2016). Effects of silver nanoparticles on primary cell cultures of fibroblasts and keratinocytes in a wound-healing model. J Appl Biomater Funct Mater 14(2): e137-142. DOI: 10.5301/ jabfm. 5000268 .

Grenier D, Chen H, Ben Lagha A, Fournier-Larente J, Morin M-P (2015). Dual Action of Myricetin on Porphyromonas gingivalis and the Inflammatory Response of Host Cells: A Promising Therapeutic Molecule for Periodontal Diseases. PLoS One 10(6): e0131758. DOI: 10.1371/journal.pone.0131758.

Hayden DM, Forsyth C, Keshavarzian A (2011). The Role of Matrix Metalloproteinases in Intestinal Epithelial Wound Healing During Normal and Inflammatory States. J Surg Res 168(2): 315-324. DOI: 10.1016/j.jss.2010.03.002.

Hou XL, Tong Q, Wang WQ, Shi CY, Xiong W, Chen J, et al. (2015). Suppression of Inflammatory Responses by Dihydromyricetin, a Flavonoid from Ampelopsis grossedentata, via Inhibiting the Activation of NF- $\mathrm{B}$ and MAPK Signaling Pathways. J Nat Prod 78(7): 1689-1696. DOI: 10.1021/acs.jnatprod.5b00275.

Hwang SH, Kim HY Zuo G, Wang Z, Lee J-Y, Lim SS (2018). Antiglycation, Carbonyl Trapping and Anti-inflammatory Activities of Chrysin Derivatives. Molecules 23(7): 1752. DOI: 10.3390/ molecules23071752.

Jang J-H, Lee SH, Jung K, Yoo H, Park G (2020). Inhibitory Effects of Myricetin on Lipopolysaccharide-Induced Neuroinflammation. Brain Sci 10(1): 32. DOI: 10.3390/brainsci10010032.

Jing N, Li X (2019). Dihydromyricetin attenuates inflammation through TLR4/NF-kappaB pathway. Open Med (Wars) 14: 719-725. DOI: 10.1515/med-2019-0083.

Juráňová J, Aury-Landas J, Boumediene K, Baugé C, Biedermann D, Ulrichová J, Franková J (2019). Modulation of Skin Inflammatory 